\title{
Electron microscopy studies of nanocrystalline zirconia
}

\author{
R RAMAMOORTHY, D SUNDARARAMAN' and S RAMASAMY* \\ Department of Nuclear Physics, University of Madras, Guindy Campus, Madras 600025 , \\ India \\ 'Metallurgy Division, Indira Gandhi Centre for Atomic Research, Kalpakkam 603 102, India
}

\begin{abstract}
Pure and yttria, calcia doped zirconia powders were prepared in nanostructured form by the method of co-precipitation by hydrolysis. As prepared and heat treated powders were characterized by $X$-ray diffraction and transmission electron microscopy. Features and variations in crystallite/particle size measurements by both XRD and TEM are discussed.
\end{abstract}

Keywords. Nanostructure; grain boundaries; lattice strains; high resolution electron microscopy.

\section{Introduction}

The size of the grains in a polycrystalline material has pronounced effects on many of its properties, the best known being the increase in strength and hardness which accompany a decrease in grain size. This dependence of properties on grain size and its internal structure is more relevant in processing of material forming operations (Cullity 1978). In the present study, XRD and TEM were used to characterize the nanocrystalline state of zirconia both in the as-prepared and annealing conditions.

\section{Experimental}

Pure and doped zirconia powders were prepared by chemical co-precipitation method (Murase and Kato 1980; Ramamoorthy et al 1995). The powder XRD of the samples were carried out by a high resolution Seifert diffractometer with $\mathrm{Cu}-\mathrm{K} \alpha 1$ radiation in the $2 \theta$ range $20-70^{\circ}$ in steps of $0-02^{\circ}$ per sec. The instrumental broadening was estimated with the XRD pattern of a standard silicon sample, and accounted properly for the crystallite size estimation by the standard procedure. The lattice parameters were determined by least squares fit of the XRD peaks and the crystallite sizes were also measured from the full width at half maximum (FWHM) of the diffraction peaks. TEM studies were carried out by using Jeol Electron Microscope JEM 2000 EXII at $200 \mathrm{kV}$. For better observations holey carbon-formvar plastic films were prepared to hold the samples. The powder particles were dispersed in isobutyl alcohol, agitated in ultrasonic cleaner for three min and a drop of the supernatent solution was put on to a 200 mesh ( $3 \mathrm{~mm}$ dia) copper grid carrying the holey carbon film.

\section{Results and discussion}

All the doped and undoped as-prepared samples showed no diffraction peaks, while the samples that were annealed at $1073 \mathrm{~K}$ for $1 \mathrm{~h}$ showed well defined diffraction peaks.

\footnotetext{
*Author for correspondence
} 


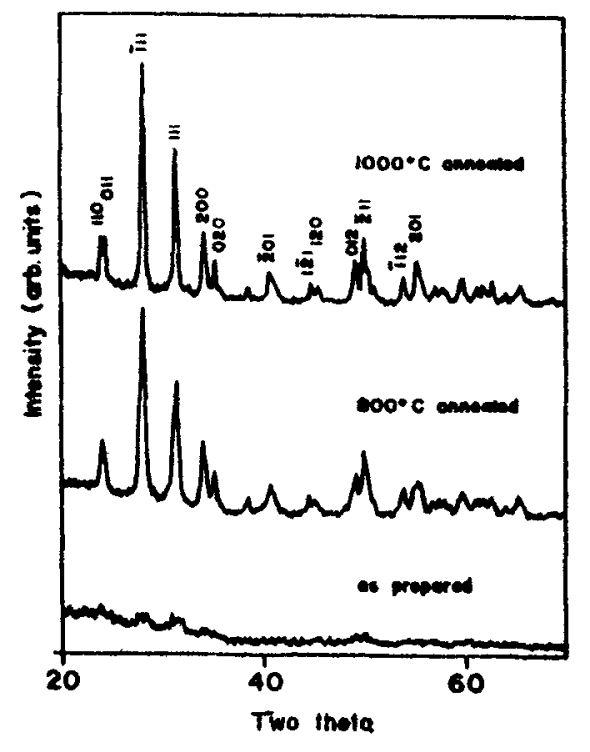

Figure 1. XRD patterns of nanocrystalline zirconia.

Samples annealed at $1273 \mathrm{~K}$, showed increased intensity of the peaks with decrease in the FWHM. Figure 1 shows the XRD pattern of as-prepared and annealed zirconia powder. The crystallite size of the samples were measured from the X-ray diffraction profiles by using the standard Scherrer's formula (Gutzov et al 1994) described as follows: It was assumed that $\mathrm{X}$-ray line broadening $\beta$ is essentially insignificant when the grain size exceeds $1000 \AA$. Then the instrumental broadening was estimated by taking X-ray diffraction for a standard sample (silicon) having the particle size more than $1000 \AA$. The actual line breadth of the sample is calculated from the formula,

$$
\beta=\left(\beta_{m}^{2}-\beta_{s}^{2}\right)^{0.5},
$$

where $\beta_{m}$ is the FWHM of the diffraction profile of the sample, and $\beta_{s}$ the FWHM of the diffraction profile of the standard.

This $\beta$ can be directly substituted in the Scherrer's formula, the particle size,

$$
t=\frac{0.9 \lambda}{\beta \cos \theta},
$$

where $\lambda$ is $X$-ray wavelength and $\theta$ the peak angle.

Using this method, the measured crystallite size increases from $8 \mathrm{~nm}$ to $17 \mathrm{~nm}$ on increasing the annealing temperature from 1073 to $1273 \mathrm{~K}$. There is no significant change in the size of the crystallite with respect to different dopants $\left(\mathrm{Y}_{2} \mathrm{O}_{3}\right.$ and $\left.\mathrm{CaO}\right)$ for the corresponding annealing treatments. However, in the calcia doped zirconia, it was found that the grain growth was limited in comparison to undoped and yttria doped zirconia samples.

In TEM, the observed particle size of the as prepared zirconia samples ranges from $50-100 \mathrm{~nm}$. For the samples annealed at temperatures 1073 and $1273 \mathrm{~K}$, we find no 


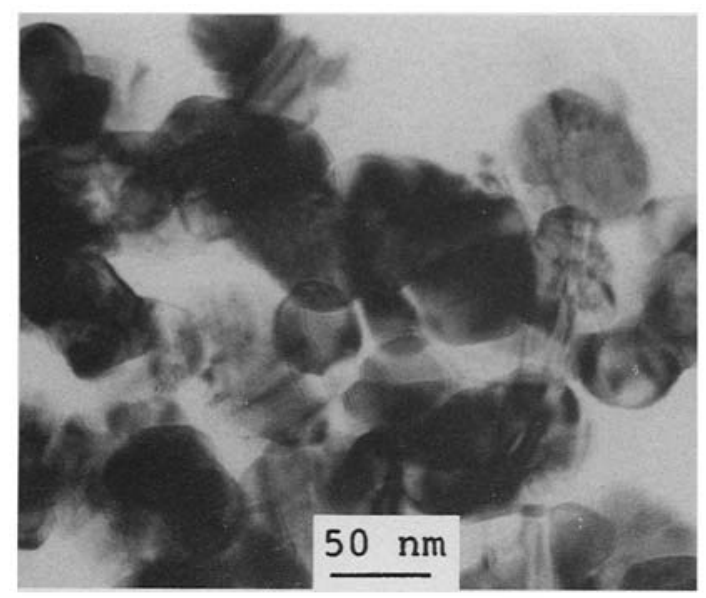

Figure 2. Microstructure of annealed $\mathrm{ZrO}_{2}$.

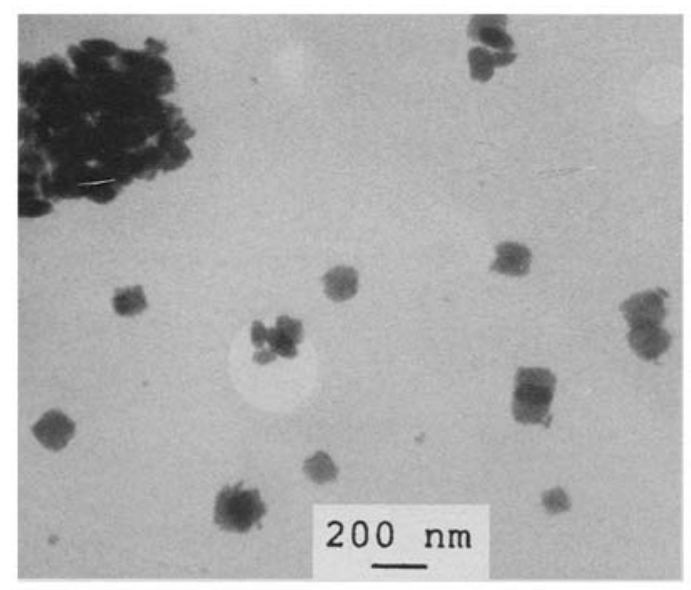

Figure 3. Particle morphology of as-prepared 3YSZ.

grain growth. The microstructure of annealed $\mathrm{ZrO}_{2}$ is shown in figure 2 . The $1273 \mathrm{~K}$ annealed sample also shows the same particle size $(50-100 \mathrm{~nm})$ as in the case of asprepared and $1073 \mathrm{~K}$ annealed samples. Further in zirconia powders doped with yttria and calcia, there is little difference in the particle size from pure zirconia powder. All the powder samples of pure and doped zirconia exhibit distinctly two characteristic particle shapes in varying amounts. The varying amounts seem to have a bearing on the dopant concentration and the crystal structure (Cahn et al 1993). The main two particle morphologies: (i) square shape and (ii) lens shape, were found to be prevalent in the various doped samples of zirconia. The as-prepared $3 \mathrm{~mol} \% \quad \mathrm{Y}_{2} \mathrm{O}_{3}-\mathrm{ZrO}_{2}$ (3YSZ) sample contains large number of lens shape particles than square shape ones as shown in figure 3 . The square shape particles are well separated and dispersed randomly, but the lens shape particles occur in agglomerates. Annealing treatments induce a shape change predominantly to a well defined round edge square shape in the various dopant concentrations that we have used in this study. The electron diffraction 


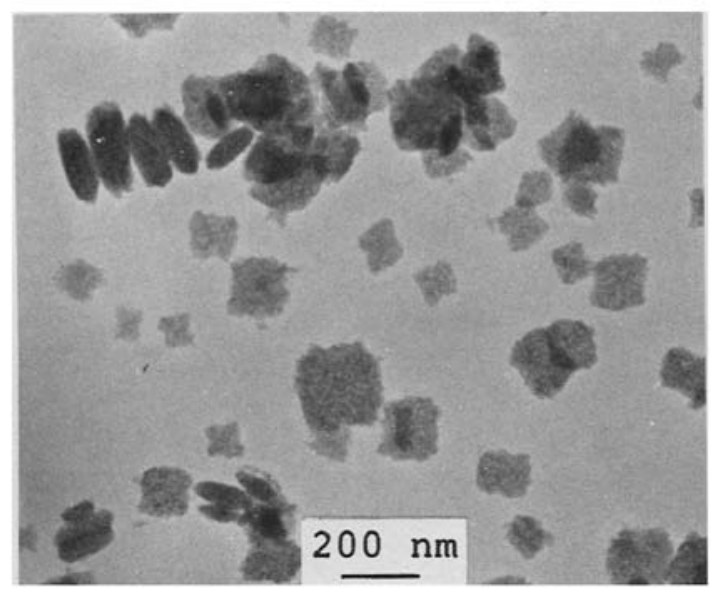

Figure 4. Particle size distribution of 12YSZ.

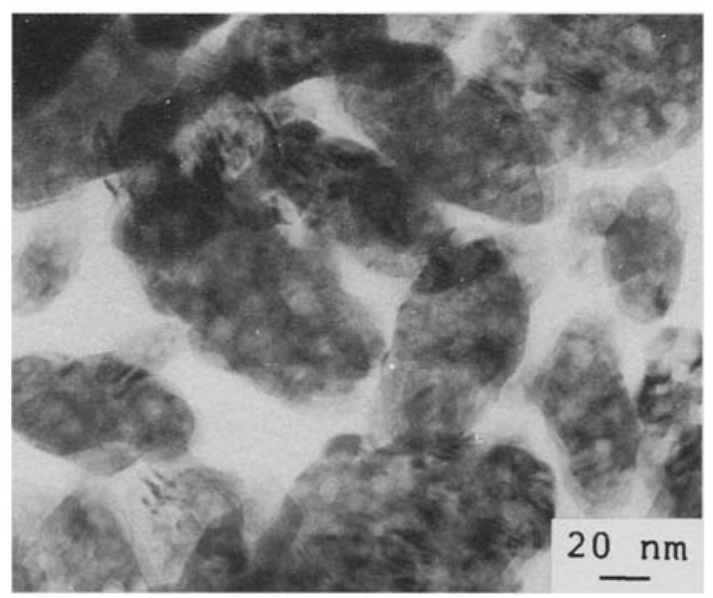

Figure 5. Internal domain structure of calcia doped zirconia.

pattern of 3 and $12 \mathrm{~mol} \% \mathrm{Y}_{2} \mathrm{O}_{3}-\mathrm{ZrO}_{2}(12 \mathrm{YSZ})$ look alike. The microstructure of annealed (12YSZ) is shown in figure 4.

A significant feature of the TEM study is that all the doped and undoped zirconia particles are aggregates or clusters of ultrafine crystallites (or domains). Each domain of the cluster is found to vary in the size range $5-15 \mathrm{~nm}$. A typical image of such a morphology is shown in figure 5 for annealed calcia doped zirconia.

The XRD patterns of as-prepared samples did not show diffraction peaks. It was believed that the sample would be amorphous in nature. But in TEM, the particle size lie in the range $50-100 \mathrm{~nm}$ (figure 2) i.e. in the nanocrystalline state. The well defined electron diffraction (ED) polycrystalline rings confirm that the sample state is not amorphous but nanocrystalline. It is to be noted that the annealed powders also showed well defined electron diffraction rings even when there is no change in particle size, except for the fact that rings are more sharper and not diffused in comparison to the as-prepared ones. The reason for the sharp diffraction pattern can be understood in 

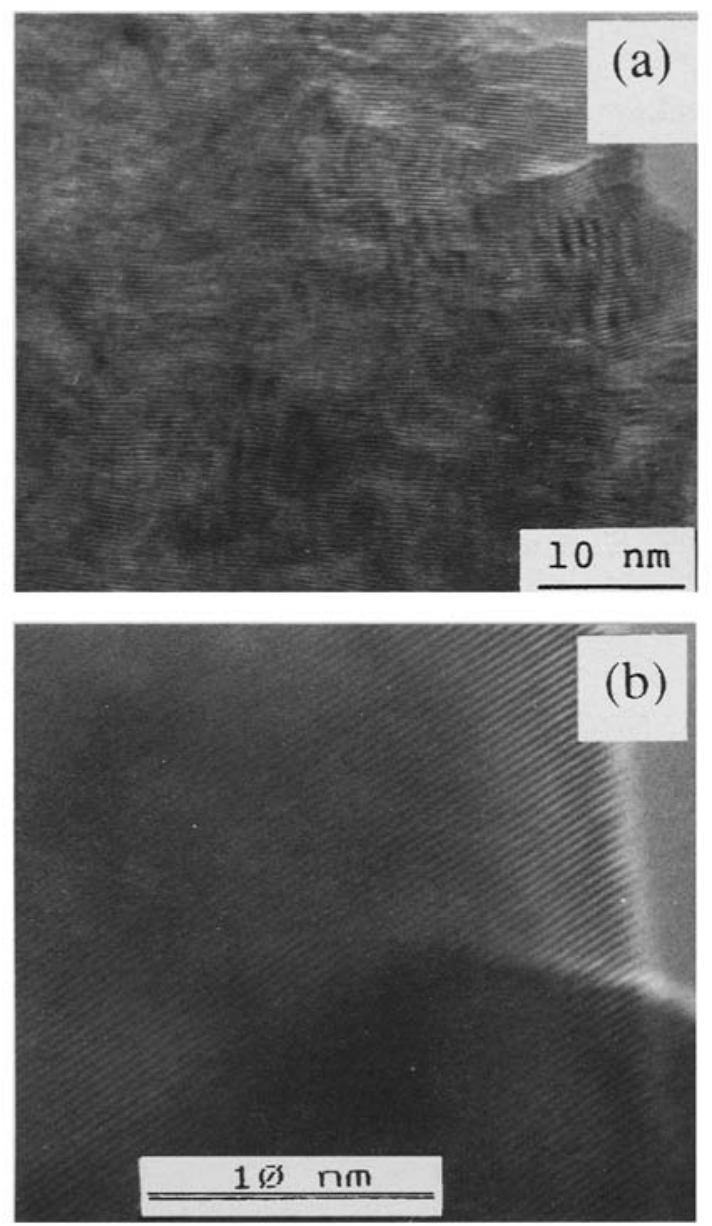

Figure 6. (a) HRTEM lattice images of as prepared 3YSZ and (b) HRTEM lattice images of 3 YSZ annealed at $1273 \mathrm{~K}$.

comparing the high resolution electron microscope (HREM) lattice images obtained for the as-prepared and annealed ones (figures $6 \mathrm{a}$ and $\mathrm{b}$ ). It is quite obvious that the absence of significant lattice strains in annealed sample but present in the as-prepared one, is responsible for the well defined ED pattern. We can understand the occurrence of XRD peaks only in the annealed condition in the following manner: (i) the predominance of excessive lattice strains that are elastic in nature, (ii) non-uniform distribution of strains resulting in random orientation of reflecting planes and (iii) increased number density of inner domain boundaries resulting in large amount of incoherent scattering, thereby decreasing the intensity of XRD peaks below the detectable limit in the as-prepared samples. In contrast, on annealing, the removal of strains result in well defined orientations of the reflecting planes. The HREM observations on the internal domain structure of the nanocrystalline particle, gave a clue to our observations. It is thus seen that there is no discrepancy between the TEM and XRD observations. 


\section{Conclusions}

(I) The absence of XRD peaks in the as-prepared samples is due to the incoherent scattering of $\mathrm{X}$-rays by the large number of grain boundaries and the lattice strains in the nanocrystalline state.(II) The lattice strains are removed when it is annealed at high temperatures. (III) Two different morphologies are seen namely the lens and square shape particles in doped samples. (IV) Each macroscopically looking particle is an aggregate of ultrafine crystallites.

\section{Acknowledgements}

Financial support by the Department of Science and Technology (DST), Government of India (Project No. III(19)/92-ET dated 28.01-1993) and UGC-SAP programmes are gratefully acknowledged.

\section{References}

Cahn R W, Haasen P and Kramer E J 1993 Materials science and technology-A comprehensive treatment (New York: VCH Publishers Inc.) Vol. 11, p. 101

Cullity B D 1978 Elements of X-ray diffraction (Addison Wesley Pub. Co. Inc.) Second ed.

Gutzov S, Ponahlo J, Lengauer C L and Beran A 1994 J. Am. Ceram. Soc. 771649

Murase Y and Kato E 1980 J. Cryst. Growth 50509

Ramamoorthy R, Viswanath R N and Ramasamy S 1995 Nanostruc. Mater. 6337 